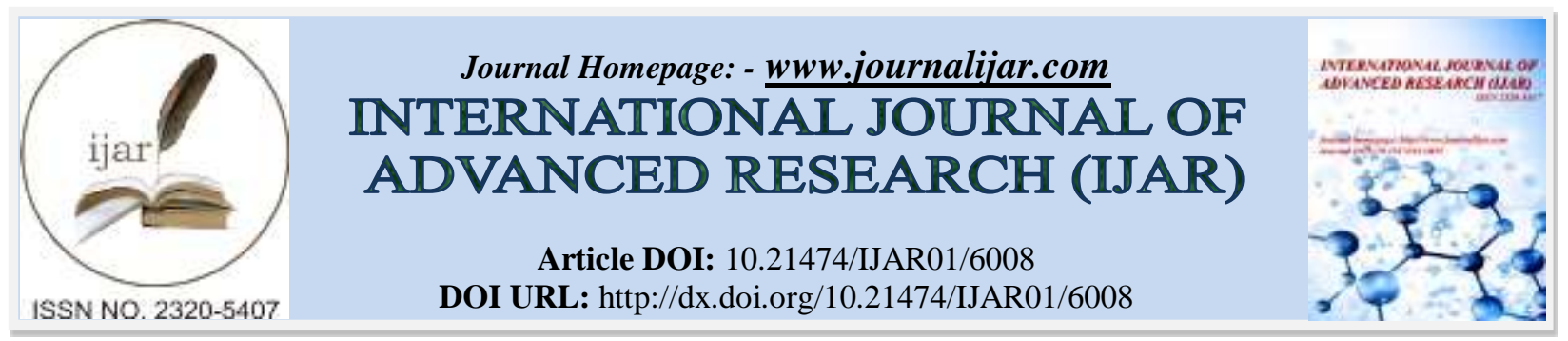

RESEARCH ARTICLE

\title{
ANTIFUNGAL ACTIVITY OF VARIOUS WEAK ORGANIC ACIDS AND THEIR SYNERGISTIC EFFECT AGAINST S. CEREVISIAE.
}

\author{
Hatice Büşra Konuk ${ }^{1}$ and Bengü Ergüden ${ }^{1}$. \\ Department of Bioengineering, Gebze Technical University, 41400, Kocaeli Turkey.
}

\section{Manuscript Info}

Manuscript History

Received: 08 October 2017

Final Accepted: 10 November 2017

Published: December 2017

Key words:-

Saccharomyces cerevisiae. Weak acids .

Antifungal activity . Synergistic effect.

\section{Abstract}

Antifungal activity of four weak organic acids against $S$. cerevisiae cells were examined. Antifungal effects of hexanoic (C6), octanoic (C8), decanoic (C10) and benzoic acids were determined through Minimum Inhibitory Concentration (MIC), and inhibition zone measurements. The most effective weak acid was decanoic acid (MIC: $0.2-0.3 \mathrm{mM}$ ) according to MIC results. In order to have some insight in the inhibition mechanism of these weak acids, their efficiency was compared with that of hydrochloric acid $(\mathrm{HCl})$ giving the same amount of drop in extracellular $\mathrm{pH}$. Results demonstrated that the inhibition of yeast cells by weak acids is not simply due to acidity, but toxic effect of the anion and the insertion of the weak acids inside the cellular membrane may play a role. Moreover, synergistic effects of weak acids were examined and it has been shown that combinations of weak acids are more effective than using weak acids alone. Thus, this research not only opens new perspectives on antifungal activity mechanisms of weak acids, but also help their usage in combination widely.

Copy Right, IJAR, 2017,. All rights reserved.

\section{Introduction:-}

Weak acids have been extensively used as preservatives in food and beverage industries against yeast including Saccharomyces cerevisiae (Lambert and Stratford, 1999; Mollapour et al., 2006; Papadimitriou et al., 2007; Ullah et al., 2012). However, yeast strains may sometimes become resistant to these preservatives, therefore their more effective usage and understanding the essence of their inhibitory mechanism is crucial.

Fatty acids (hexanoic/caproic, octanoic/caprylic, decanoic/capric) are carboxylic acids characterized by the presence of a carboxyl group (-COOH) at one end and a methyl group $\left(-\mathrm{CH}_{3}\right)$ at the other end. Octanoic and decanoic acids are well known as fermentation inhibitors produced by yeasts during alcoholic fermentation (Legras et al., 2010).

Antifungal effect of these fatty acids against S. cerevisiae has been reported (Bergsson et al., 2001; Cabral et al., 2001; Kumar et al., 2011). The toxicity of short to medium chain fatty acids is correlated with their lipophilic properties (Tenreiro et al., 2002) and their key effect was proposed to be the cell membrane damage (Desbois and Smith, 2010; Ricke, 2003). These lipophilic weak acids can cross the plasma membrane by passive diffusion and dissociate their liposoluble form in the neutral cytosol at higher internal $\mathrm{pH}$, leading to decrease of the intracellular pH and accumulation of toxic anion (Cabral et al., 2001; Legras et al., 2010; Viegas and Sá-Correia, 1997).

Corresponding Author:- Bengü Ergüden.

Address:- Department of Bioengineering, Gebze Technical University, 41400, Kocaeli Turkey. 
Benzoic acid as a weak organic acid is significant for preventing microbial spoilage (Hazan et al., 2004; Kresnowati et al., 2008) and there are number of theories that explain the antifungal properties of weak acid, but the exact mechanism is still unknown (Ullah et al., 2012). Several studies have supported the notion that weak organic acids function through disruption of membrane organization through acidity. (Hatzixanthis et al., 2003; Holyoak et al., 1999). However, another study indicated that this acidification does not correlate with growth inhibition (Bracey et al., 1998), and therefore, acidification does not alone represent antifungal mechanism of benzoic acid.

In this study, we carried out an investigation of yeast response to hexanoic, octanoic, decanoic and benzoic acid and their combinations. Due to our continuing interest on the mode of action of various chemicals on yeast membranes, we set out to unearth the possible membrane dependent action and to show that the inhibition of yeast cells is not only due to the acidity of weak acids.

\section{Materials and methods:-}

Minimum inhibitory concentration (MIC) measurement:-

S. cerevisiae YPH499 strain was cultured overnight at $25^{\circ} \mathrm{C}$ in YPD broth. Test strain was suspended in YPD to give a final density of $1 \times 10^{6} \mathrm{CFU} / \mathrm{mL}$ (Kumar Tyagi et al., 2014). Weak acids dissolved in DMSO were diluted to the mentioned concentrations in 24-well microtiter plate and then $S$. cerevisiae cells were added to each well. Plates were incubated for $48 \mathrm{~h}$ at $25^{\circ} \mathrm{C}$ with shaking. After incubation, MIC values were determined as the lowest concentration of acids by detecting the appearance of any visible growth of yeasts (Kumar Tyagi et al., 2014; Shimazaki et al., 2016).

\section{Inhibition zone measurement:-}

The antifungal activities of weak acids against YPH499 strain was determined by employing the standard disks diffusion method (Bauer et al., 1966; Rattanachaikunsopon and Phumkhachorn, 2010). In this method, $500 \mu \mathrm{L}$ of fresh culture of YPH499 was spread on the YPD agar media plates. $5 \mathrm{~mm}$ diameter wells were opened. Weak acids were diluted in DMSO at $10 \%$ concentration and $55 \mu \mathrm{L}$ of acids were filled into each well. Plates were incubated at $25^{\circ} \mathrm{C}$ for 48 hours. After incubation, the diameters of the inhibition zones were measured in centimeter.

\section{Extracellular pH measurement :-}

YPH499 strain was cultured overnight at $25^{\circ} \mathrm{C}$ in $20 \mathrm{~mL}$ of YPD broth. After incubation, the yeast cells were centrifuged at $3200 \mathrm{rpm}$ for $5 \mathrm{~min}$ and pellet was washed twice with sterilized $\mathrm{dH}_{2} \mathrm{O}$. The pellet was then resuspended in sterilized $\mathrm{dH}_{2} \mathrm{O}$. About $50 \mathrm{mg}$ wet weight of yeast cells were used for each experiment (Gášková et al., 2013; Wang et al., 2015). The weak acids dissolved in DMSO were added to the above resuspended solution. $\% 2$ glucose (zero point) and weak acids (18th min) were manually injected to the mentioned final concentrations. Extracellular $\mathrm{pH}$ was recorded at the indicated times with an HI 98127 water proof $\mathrm{pH}$ meter (HANNA, USA).

\section{Statistical Analyses:-}

MIC, inhibition zone assay, extracellular $\mathrm{pH}$ and extracellular conductivity measurements were repeated several times. Averages of all experimental groups were calculated and standard deviations were determined using Microsoft Excel.

\section{Results and Discussion:-}

S. cerevisiae is one of the common yeasts associated with food spoilage and acquired resistance to weak acids by increasing expression of the ABC (ATP-binding cassette) transporter, Pdr12p, which promotes the active leakage of these compounds (Hatzixanthis et al., 2003; Holyoak et al., 1999; Mollapour et al., 2006). Control of fungal spoilage and growth is a critical problem for food industry, medical field and other areas.

After a thorough search of the literature we uncovered that the information on the antifungal activity against $S$. cerevisiae using MIC and inhibition zone assay was limited. More importantly experimental data reported in the literature are based on studies with different experimental conditions preventing a simple comparison of the data with each other. Thus, at the beginning of our studies we set out to determine the MIC and inhibition zone values as a measurement of antifungal activity of weak acids (Table 1). 
Table 1:- Antifungal activity of weak acids presented as zones of inhibition and MIC values. Weak acids were mixed with DMSO to increase solubility.

\begin{tabular}{|c|c|c|c|}
\hline$\#$ & Weak acids & Inhibition zones $(\mathbf{c m}) *$ & MIC (mM) \\
\hline 1 & hexanoic acid & $3.3 \pm 0.3$ & $4.0-8.0$ \\
\hline 2 & octanoic acid & $2.7 \pm 0.2$ & $1.5-2.5$ \\
\hline 3 & decanoic acid & $2.9 \pm 0.3$ & $0.2-0.3$ \\
\hline 4 & benzoic acid & $2.0 \pm 0.2$ & $4.0-8.0$ \\
\hline
\end{tabular}

* $10 \%$ of weak acid was used.

According to MIC results, decanoic acid $(0.2-0.3 \mathrm{mM})$ is more toxic than octanoic and other weak acids. On the contrary, hexanoic acid ( $3.3 \pm 0.3)$ is most effective weak acid in inhibition zone results. Our results supported the findings of previous studies (Alexandre et al., 1996; Viegas et al., 1989), where decanoic acid was reported to be more toxic than octanoic acid since it is more liposoluble causing an increase in cell permeability (Alexandre et al., 1996; Bergsson et al., 2001; Kumar et al., 2011).

On the other hand, addition of weak organic acids, caused a sudden decrease of extracellular $\mathrm{pH}$ (Fig 1). When yeast cells were treated with $\mathrm{HCl}$ in the as much necessary amount to provide the same $\mathrm{pH}$ drop (i.e. up to $10^{-3} \mathrm{M}$ ) (Fig 2), they continued to survive (Table 2). Thus inhibitory effect of weak acids does not occur solely through acidity, toxic effects of the anion and the increased permeability of the cell membrane due to the lipophilicity of the weak acid must be the underlying phenomenon in antifungal activity.
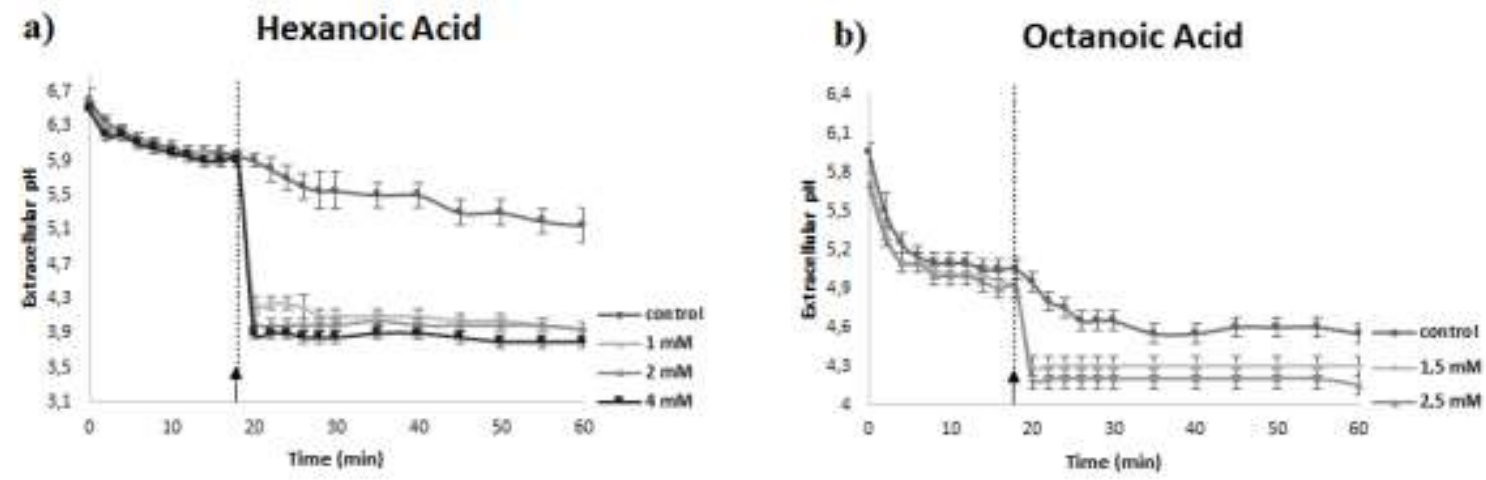

c)

Decanoic Acid

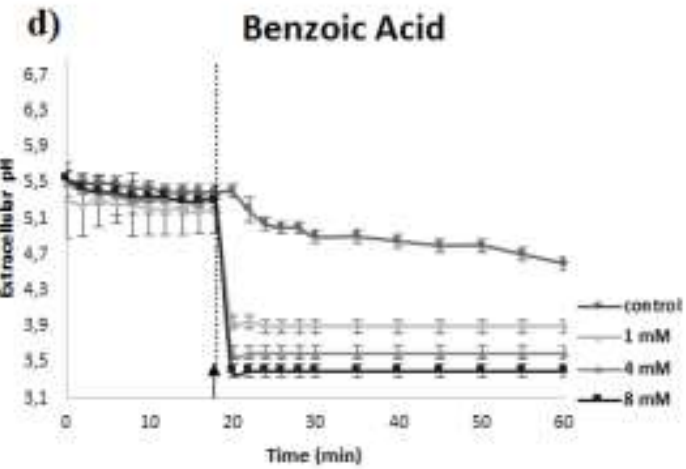

Fig 1:- Effects of weak acids on extracellular $\mathrm{pH}$ of $S$ cerevisiae in glucose-induced medium. The arrows indicate the time of addition of a) hexanoic acid, b) octanoic acid, c) decanoic acid and d) benzoic acid. Horizontal axis time (minute); vertical axis - extracellular $\mathrm{pH}$. 


\section{Hydrochloric Acid}

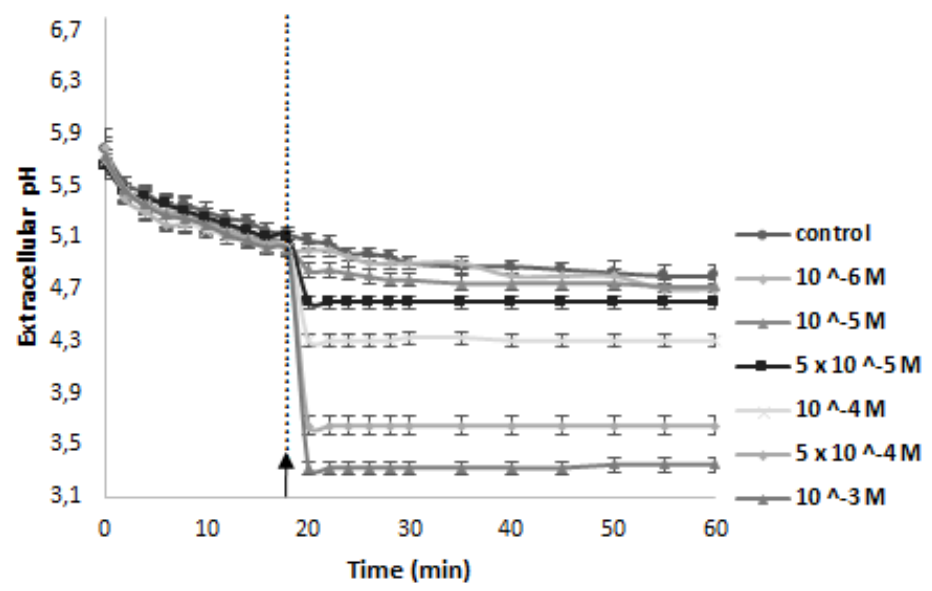

Fig 2:- Effects of $\mathrm{HCl}$ in various concentrations which provide the same $\mathrm{pH}$ drop as weak acids on extracellular $\mathrm{pH}$ of $S$ cerevisiae in glucose-induced medium. The arrows indicate the time of addition of $\mathrm{HCl}$.

Table 2:- Viability of S. cerevisiae cells upon exposure to $\mathrm{HCl}, N D$ : not determined.

\begin{tabular}{|c|c|c|c|}
\hline$\#$ & HCl concentration (M) & Cell viability & Final extracellular pH \\
\hline 1 & $10^{-4}$ & + & 4.30 \\
\hline 2 & $10^{-3}$ & + & 3.35 \\
\hline 3 & $10^{-2}$ & + & $\mathrm{ND}$ \\
\hline 4 & $10^{-1}$ & - & $\mathrm{ND}$ \\
\hline
\end{tabular}

These results suggested us that the combination of these weak acids may enhance their antifungal activity and we set out to investigate the synergistic effect of their combinations. Although the inhibition effects of weak acids have been investigated before (Alexandre et al., 1996; Hazan et al., 2004; Jarboe et al., 2011; Legras et al., 2010), the antifungal activity of their combinations has not been reported against $S$. cerevisiae. Thus, various combinations of acids were prepared and viability of yeast cells in the presence of these mixtures were determined (Table 3 ).

Table 3:- Synergistic effects of weak acids against S. cerevisiae cells. Weak acids were mixed with DMSO to increase solubility.

\begin{tabular}{|c|c|c|c|c|c|c|c|c|c|c|c|c|c|}
\hline $\mathrm{a}$ & & \multicolumn{5}{|c|}{ Hexanoic Acid } & $\mathrm{d}$ & \multicolumn{6}{|c|}{ Benzoic Acid } \\
\hline & $\mathbf{m M}$ & 0 & 1 & 2 & 4 & 8 & & $\mathbf{m M}$ & 0 & 1 & 2 & 4 & 8 \\
\hline \multirow{5}{*}{ لَّ } & 0 & + & + & + & + & - & \multirow{5}{*}{ 递 } & 0 & + & + & + & + & - \\
\hline & 0.5 & + & + & + & - & - & & 1 & + & + & + & + & - \\
\hline & 1 & + & + & - & - & - & & 2 & + & + & + & - & - \\
\hline & 2,5 & - & - & - & - & - & & 4 & + & + & - & - & - \\
\hline & & & & & & & & 8 & - & - & - & - & - \\
\hline b & \multicolumn{6}{|c|}{ Octanoic Acid } & $\mathrm{e}$ & \multicolumn{6}{|c|}{ Benzoic Acid } \\
\hline \multirow{5}{*}{ 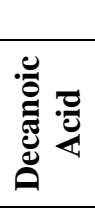 } & $\mathbf{m M}$ & 0 & 0.5 & 1 & 1.5 & 2.5 & & $\mathbf{m M}$ & 0 & 1 & 2 & 4 & 8 \\
\hline & 0 & + & + & + & + & - & \multirow{4}{*}{ لِّ } & 0 & + & + & + & + & - \\
\hline & 0.1 & + & + & + & - & - & & 0.5 & + & + & + & + & - \\
\hline & 0.2 & + & - & - & - & - & & 1 & + & + & - & - & - \\
\hline & 0.3 & - & - & - & - & - & & 2.5 & - & - & - & - & - \\
\hline c & \multicolumn{6}{|c|}{ Hexanoic Acid } & $\mathrm{f}$ & \multicolumn{6}{|c|}{ Benzoic Acid } \\
\hline \multirow{5}{*}{ 递 } & $\mathbf{m M}$ & 0 & 1 & 2 & 4 & 8 & \multirow{5}{*}{ 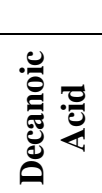 } & $\mathbf{m M}$ & 0 & 1 & 2 & 4 & 8 \\
\hline & 0 & + & + & + & + & - & & 0 & + & + & + & + & - \\
\hline & 0.1 & + & + & + & + & - & & 0.1 & + & + & + & + & - \\
\hline & 0.2 & + & + & + & - & - & & 0.2 & + & + & - & - & - \\
\hline & 0.3 & - & - & - & - & - & & 0.3 & - & - & - & - & - \\
\hline
\end{tabular}


Our results demonstrate that it is possible to achieve same inhibitory effect using less amounts of combinations of these weak acids. In general combinations of fatty acids within themselves are more effective than combinations with benzoic acid (Compare for example Table 3b with 3e and 3f). In particular octanoic acid-decanoic acid combination is the most powerful one.

\section{Conclusion:-}

In conclusion, we have shown that inhibition of yeast cells by weak organic acids is not associated with their acidity. More importantly, using weak acids in combinations increase their potency as antifungal agents, enabling their minimal amount of usage as preservatives. These results enrich our knowledge about the mechanism of action of weak acids against $S$. cerevisiae cells and help us widen their usage in food, cosmetic and pharmaceutical industries.

\section{References:-}

1. Alexandre, H., Mathieu, B., Charpentier, C. (1996): Alteration in membrane fluidity and lipid composition, and modulation of H+-ATPase activity in Saccharomyces cerevisiae caused by decanoic acid. Microbiology, 142:469-475.

2. Bauer, A., Kirby, W., Sherris, J.C., Turck, M. (1966): Antibiotic susceptibility testing by a standardized single disk method. Am. J. Clinical Pathol., 45:493.

3. Bergsson, G., Arnfinnsson, J., Steingrímsson, Ó., Thormar, H. (2001): In vitro killing of Candida albicans by fatty acids and monoglycerides. Antimicrobial Agents and Chemotherapy, 45:3209-3212.

4. Bracey, D., Holyoak, C., Coote, P. (1998): Comparison of the inhibitory effect of sorbic acid and amphotericin B on Saccharomyces cerevisiae: is growth inhibition dependent on reduced intracellular pH? J.of Appl. Microbiol., 85:1056-1066.

5. Cabral, M.G., Viegas, C.A., Sá-Correia, I. (2001): Mechanisms underlying the acquisition of resistance to octanoic-acid-induced-death following exposure of Saccharomyces cerevisiae to mild stress imposed by octanoic acid or ethanol. Arch. of Microbiol., 175:301-307.

6. Desbois, A.P., Smith, V.J. (2010): Antibacterial free fatty acids: activities, mechanisms of action and biotechnological potential. Appl. Microbiol. Biotech., 85:1629-1642.

7. Gáśková, D., Plášek, J., Zahumenský, J., et al. (2013): Alcohols are inhibitors of Saccharomyces cerevisiae multidrug-resistance pumps Pdr5p and Snq2p. FEMS Yeast Res., 13:782-795.

8. Hatzixanthis, K., Mollapour, M., Seymour, I., et al. (2003): Moderately lipophilic carboxylate compounds are the selective inducers of the Saccharomyces cerevisiae Pdr12p ATP binding cassette transporter. Yeast, 20:575585 .

9. Hazan, R., Levine, A., Abeliovich, H. (2004): Benzoic acid, a weak organic acid food preservative, exerts specific effects on intracellular membrane trafficking pathways in Saccharomyces cerevisiae. Appl. Env. Microbiol., 70:4449-4457.

10. Holyoak, C.D., Bracey, D., Piper, P.W., Kuchler, K., Coote, P.J. (1999): The Saccharomyces cerevisiae weakacid-inducible $\mathrm{ABC}$ transporter Pdr12 transports fluorescein and preservative anions from the cytosol by an energy-dependent mechanism. J. Bacteriol., 181:4644-4652.

11. Jarboe, L.R., Liu, P., Royce, L.A. (2011): Engineering inhibitor tolerance for the production of biorenewable fuels and chemicals. Curr. Op. Chem. Eng., 1:38-42.

12. Kresnowati, M., van Winden, W., van Gulik, W., Heijnen, J. (2008): Energetic and metabolic transient response of Saccharomyces cerevisiae to benzoic acid. The FEBS J., 275:5527-5541.

13. Kumar, A., Singh, S., Jain, S., Kumar, P. (2011): Synthesis, antimicrobial evaluation, QSAR and in silico admet studies of decanoic acid derivatives. Act. Pol. Pharm., 68:191-204.

14. Kumar Tyagi, A., Bukvicki, D., Gottardi, D., et al. (2014): Eucalyptus essential oil as a natural food preservative: in vivo and in vitro antiyeast potential. BioMed Res. Int., 2014.

15. Lambert, R., Stratford, M. (1999): Weak-acid preservatives: modelling microbial inhibition and response. J. Appl. Microbiol., 86:157-164.

16. Legras, J., Erny, C., Le Jeune, C., et al. (2010): Activation of two different resistance mechanisms in Saccharomyces cerevisiae upon exposure to octanoic and decanoic acids. Appl. Env. Microbiol., 76:7526-7535.

17. Mollapour, M., Phelan, J.P., Millson, S.H., Piper, P.W., Cooke, F.T. (2006): Weak acid and alkali stress regulate phosphatidylinositol bisphosphate synthesis in Saccharomyces cerevisiae. Biochem. J., 395:73-80.

18. Papadimitriou, M.N., Resende, C., Kuchler, K., Brul, S. (2007): High Pdr12 levels in spoilage yeast (Saccharomyces cerevisiae) correlate directly with sorbic acid levels in the culture medium but are not 
sufficient to provide cells with acquired resistance to the food preservative. Int. J. Food Microbiol., 113:173179.

19. Rattanachaikunsopon, P., Phumkhachorn, P. (2010): Lactic acid bacteria: their antimicrobial compounds and their uses in food production. Annals Biol. Res., 1:218-228.

20. Ricke, S. (2003): Perspectives on the use of organic acids and short chain fatty acids as antimicrobials. Poultry Sci., 82:632-639.

21. Shimazaki, A., Sakamoto, J.J., Furuta, M., Tsuchido, T. (2016): Antifungal Activity of Diglycerin Ester of Fatty Acids against Yeasts and Its Comparison with Those of Sucrose Monopalmitate and Sodium Benzoate. Biocontrol Sci., 21:123-130.

22. Tenreiro, S., Nunes, Pc.A., Viegas, C.A., et al. (2002): AQR1 gene (ORF YNL065w) encodes a plasma membrane transporter of the major facilitator superfamily that confers resistance to short-chain monocarboxylic acids and quinidine in Saccharomyces cerevisiae. Biochem. Biophys. Res. Commun., 292:741-748.

23. Ullah, A., Orij, R., Brul, S., Smits, G.J. (2012): Quantitative analysis of the modes of growth inhibition by weak organic acids in Saccharomyces cerevisiae. Appl. Env. Microbiol., 78: 8377-8387.

24. Viegas, C.A., Rosa, M.F., Sá-Correia, I., Novais, J.M. (1989): Inhibition of yeast growth by octanoic and decanoic acids produced during ethanolic fermentation. Appl. Env. Microbiol., 55: 21-22.

25. Viegas, C.A., Sá-Correia, I. (1997): Effects of low temperatures (9-33 C) and pH (3.3-5.7) in the loss of Saccharomyces cerevisiae viability by combining lethal concentrations of ethanol with octanoic and decanoic acids. Int. J. Food Microbiol., 34:267-277.

26. Wang, Y., Zeng, X., Zhou, Z., et al. (2015): Inhibitory effect of nerol against Aspergillus niger on grapes through a membrane lesion mechanism. Food Control, 55:54-61. 